\title{
How Human Inspired Learning Enhances the Behavior of Autonomous Agents
}

\author{
Martin Fittner*, Christian Brandstaetter \\ Institute of Computer Technology at Vienna University of Technology, Gußhausstraße 27-29, 1040 Vienna. \\ * Corresponding author. Email: fittner@ict.tuwien.ac.at \\ Manuscript submitted January 15, 2017; accepted May 10, 2017. \\ doi: 10.17706/jcp.13.2.154-160
}

\begin{abstract}
An autonomous agent must deal with unforeseen situations that can't be preprogrammed. Therefore, the agent has to make its own experiences, solutions and valuations to situations, actions and objects to be able to enhance previous actions and avoid repeating wrong actions. On the basis of the existing cognitive architecture Simulation of Mental Apparatus and Applications (SiMA) at the Institute of Computer Technology (ICT) an bionically inspired attempt of learning should be implemented in functional model of the human mind which is then used in a multi-agent simulation showing how bionically inspired cognitive architectures can get extended by learning. Due to the attempt in the project SiMA the learning function has to fit in the psychoanalytic model and therefore it has to be compatible with the way of learning that human beings do. This might also help to get a little bit closer to the understanding of how the human mind manipulates memory to show these until now unreached cognitive abilities.
\end{abstract}

Key words: Learning, artificial general intelligence, simulation of mental apparatus and applications (sima), artificial recognition system (ars), cognitive architectures, cognitive automation, psychoanalytically inspired ai, software agents.

\section{Introduction}

How a human being learns is one of the fascinating questions that can't be solved by any science discipline until now. To get a little bit closer to that answer, the topic should be discussed together within the project Simulation of Mental Apparatus and Applications (SiMA) which is a functional model of the human mind. To cope with the rising demands in building automation, the project SiMA was founded by Dietmar Dietrich [1]. It introduces a bionic approach and uses the human mind as an archetype for cognitive processing. Another impulse why this project was introduced was the disappointing progress in artificial intelligence on generating human-like behavior and successfully fulfill the task of bringing somebody a glass of water [2].

The project uses a multi-agent simulation environment based on the MASON [3] framework to prove the correctness of the functional model and its underlying concepts. The functional model itself was developed together with psychoanalysts and inspired from the Psychoanalytic Model of the human mind To achieve this goal, we use the best holistic functional model [4] of the human mind that was available, the Metapsychology from Sigmund Freud [5]. The big hypotheses in this basic research project is that the metapsychology offers a valid functional model of the human mind. This hypothesis has been evaluated by [6], [7] that stated psychoanalysis fulfills the needed criteria of one holistic model of the mind.

Until now learning is a missing feature in the project SiMA. In this paper, we start with the first deliberations of how to implement learning in a functional model of the human mind and a small scenario 
will be simulated that includes learning abilities.

\section{Background and Related Works}

There is a huge number of algorithms for machine learning (reinforced learning, deep learning, neural networks, ...) which are used successfully for different technical problems. Their strength lies in solving a particular technical problem better than other solutions. The algorithms were refined over the years and are now more successfully than before and used in a lot of technical solutions. But they still lack of an overall general learning ability that can be compared to the way a human being learns maybe also because they are very limited in its functionality. For example, a chess computer can normally play any other board game just by changing some parameters in the program that are related to the rules of the game. Therefore, they don't fit as an overall solution into our holistic functional model of the human mind. But there are still other projects in the area of artificial general intelligence that should be shortly discussed here by picking LIDA (Learning Intelligent Distribution Agent) as a representative concerning the similarity to SiMA [8] and the learning functions.

LIDA combines recent theories of the mind in a cognitive architecture [9]. It is an extension of the IDA model with the feature that it is capable of learning from its experiences. The model is based primarily on global workspace theory (GWT) which follows the connectionist theory and psychological and neurobiological theories of the role of consciousness in cognition [9]. There are concepts used like emotions and feelings for the evaluation of situations. Perceptual learning and episodic learning are already implemented in LIDA, and other learning functions are conceptually included in the framework. In the concept of LIDA new information is learned only when it becomes conscious.

\section{The SiMA Project}

SiMA follows a bionic approach and uses the model of the metapsychology as a framework for building a functional model of the human mind. The Model is divided into three layers where the lowest on is the neural layer which handles signals from sensors and actors. These signals are transferred into neurosymbols in the 2nd layer which was defined and described by [10]. The third layer constitutes the mind which was the starting point of SiMA. Until now layer 1 (L1) and 2 (L2) are only represented as dummies, so our focus lies on the functionality of the mind (L3).

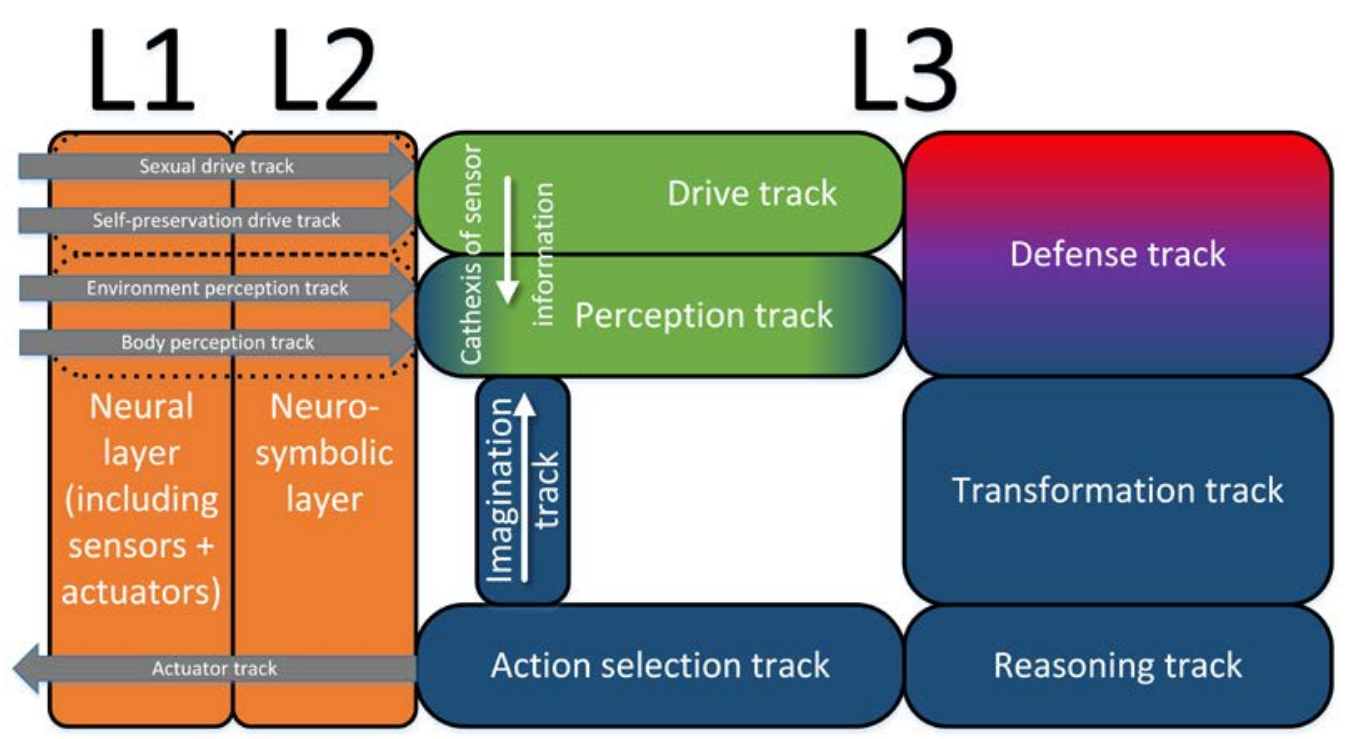

Fig. 1. Functional model of SiMA in an abstract view [11]. 
Layer 3 of the model is further divided into unconscious data processing and conscious or preconscious data processing. An abstract view of the model is shown in Fig. 1 where the drive, the perception and the defense track resides in the unconscious data processing part of the model. The functions were broken down to the lowest level which results in a model with 43 functions. For the integration of learning only a piece of the model was enhanced with the functionality of storing new information. In particular, two functions of the perception track are modified to get first results in a simulation run.

\section{A Human-Inspired Learning Model}

The big parts of the model are the memories, where data can be stored and the reasons for storing data in one of the memories. These reasons are calculated valuations that significantly deviate from already stored data in the long-term memory (LTM). In the next sections, the memory model and the motivation to store new or adapted data should be discussed.

\subsection{Memory in the SiMA Project}

The memory storage model that is used in SiMA corresponds to the multi storage model of Atkinson und Shiffrin [12]. A sensory memory that is used there is not needed in SiMA because of the undeveloped hardware layer the data that is handed over to the layer 3. But as the data are static for a whole cycle through all functions of the model it can be seen as matchable to a sensory memory. Further, SiMA is equipped with a LTM and a short-term memory (STM). Until now the LTM transfer all data from an ontology (only used as structured data storage without production rules) to the functions. Then they are used for decision making, but experiences are not stored up to now (see red arrow in Fig. 2).

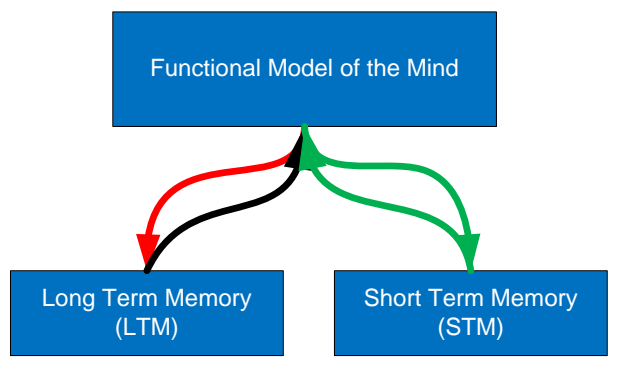

Fig. 2. Memory model in SiMA.

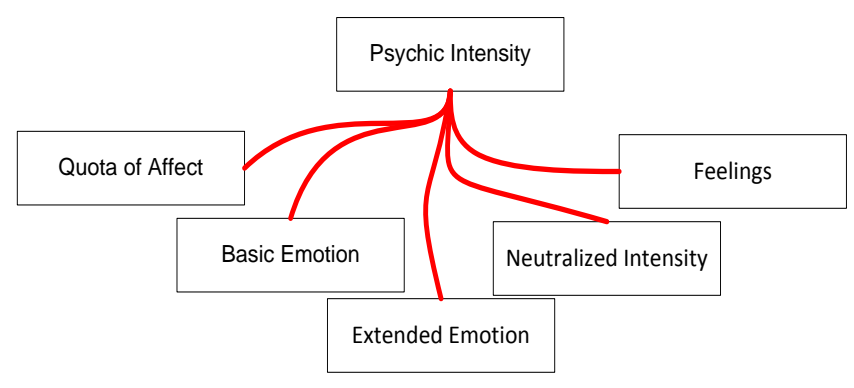

Fig. 3. Psychic intensity and its components.

Since biologic functions always rely on a useful idea, we might have a look at the service of memory in the human brain and how it can enhance our functions. The main functionality of the STM can be seen as a storage for helping the functions to get a better solution than without it. So, important data can't be collected together only with perception at once. They have to be parked to bring them in relation to other perceptible data. STM is then the service to make better decisions by enhancing the perception in the current moment. The LTM is needed to store the outcome of such situations with a filter regarding their importance and the exposure time. A detailed description of the memory in SiMA can be found in [13].

The aim of introducing learning in the project SiMA is to develop a holistic model for learning. So the focus lies on an overall learning ability within the model and not on specialized situations or tasks. In this manner, a functionality that can store new data (similar to the consolidation) should be implemented first. In further implementations, adaption of already known data will follow.

\subsection{Psychic Intensity}

The term psychic intensity was defined in the SiMA project and summarizes an evaluated term for 
tensions of the body. According to [13] "In the SiMA project, psychic intensity is used as an umbrella term for all valuation quotas...Valuation ultimately serves to prioritize actions in order to mediate between the demands of the outside world (the own body and the environment) and the inner (psychic) needs (e.g. satisfying psychic and physiological needs within the environment, or adapting (psychic) wishes to the external circumstances)". Psychic intensity contains 5 different valuations which are: quota of affect, basic emotions, extended emotions, neutralized intensity and feelings.

All decisions that are made by the model are based on these 5 valuations and therefore they are the first candidates to store them for proper reactions in similar situations. In this paper, the quota of affect is used as the first parameter that can be manipulated in the memory. It is a measure of the tension of the current drives like low blood sugar. In contrast to the other parameters of psychic intensity the quota of affect has a direct relation to a current need of the body.

In further implementations, emotions should be integrated into the learning abilities of the model. Emotions can represent the agent's current state in a physical and psychological manner. That data can be used to categorize similar situations and reactions in that particular situation under integration of a stored internal state of the agent.

\subsection{Exemplary Case}

The exemplary case was introduced in the SiMA project [14]. It describes in a narrative form a particular case that demonstrates assumptions in an exemplary form e.g. decision making in a concrete internal and external situation. In this paper, the exemplary case should show how learning of new experiences is done. The exemplary case is further divided into simulation cases that describe a particular situation.

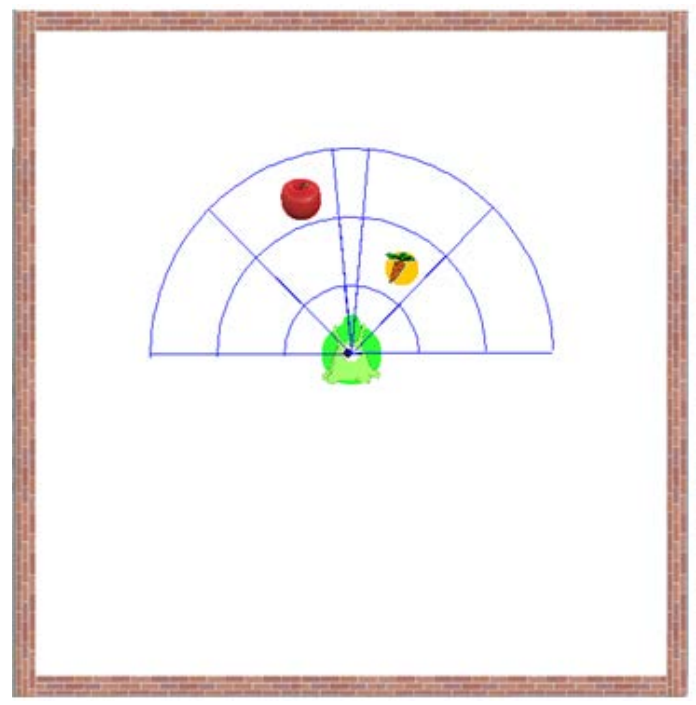

Fig. 4. Starting point of the simulated scenario.

The starting point of the first simulation case (Fig. 4) shows the green agent, which is parameterized as a hungry person. He is placed together with two food sources in a closed room. The memories of the agent contain both objects and also plans how to eat them but lack of an experience which drives they satisfy. That satisfaction value should be learned by the agent. This very simple looking scenario hides a lot of internal processes (see Fig. 1) that the agent will process to come to a decision that is executed. Therefore, inspectors are used to show the internal state, evaluation of data, decision-making and memories.

The standard procedure in SiMA was a setup of such a situation and the production of different outcomes in the identical situation with the change of behavioral determinants. These determinants were manually edited before the simulation and evaluated during and after the simulation. We distinguish four groups of 
determinants: the environmental situation, the agent's internal state (drives and emotions), personality factors (as simplifications of memories and body functionalities) and the agent's memories (experience).

With the integration of a learning ability, the manual edition isn't needed anymore to generate different behavior. The agent now has to make and store its own experiences in a situation. To show this behavior, all determinants are not manipulated manually. Only the memory is edited by the agent itself. Depending on the parameters of the objects (apple and carrot) the agent chooses the object that can satisfy its drives. In the simulation case, the carrot has less ability to satisfy the hunger of the agent than the apple.

\section{Simulation}

The current implementation state is still at its beginning but first simulation results are ready to be discussed here. The first simulation case which was described above was executed and showed the expected behavior from section 4.3. Depending on the drive satisfaction ability of the objects the agent chooses the objects that satisfies its needs after a learning phase which delivers the valuations for the next simulation run. So the simulation is divided into two runs. The first one shows what the agent will do when he can't learn out of experiences. Then the behavior of the agent will be the same in further iterations.

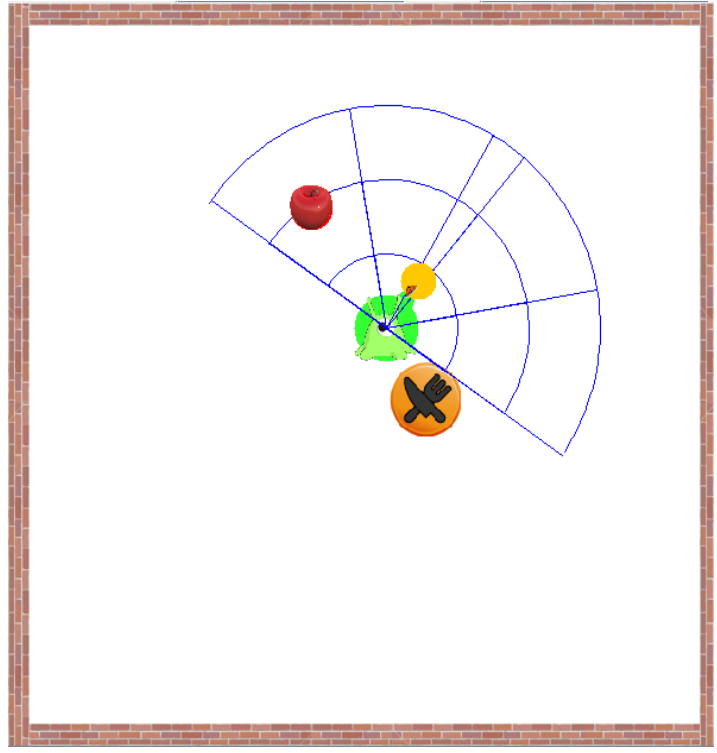

Fig. 5. Learning phase.

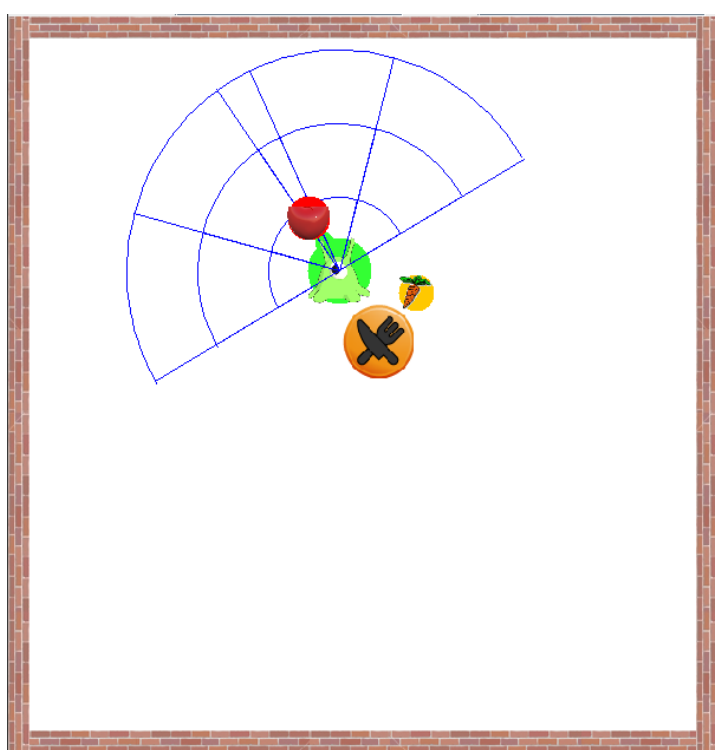

Fig. 6. Behavior after learning in 2nd run.

Table 1. Memories of Quota of Affect during Simulation

\begin{tabular}{l|lll}
\hline \hline Objects & $1^{\text {st }}$ run start & $1^{\text {st }}$ run end & $2^{\text {nd }}$ run end \\
\hline Cake & High drive satisfaction & High drive satisfaction & High drive satisfaction \\
Apple & None (same as cake) & None (same as cake) & Medium drive satisfaction \\
Carrot & None (same as cake) & Low drive satisfaction & Low drive satisfaction \\
\hline \hline
\end{tabular}

When the agent is equipped with a learning functionality, the first simulation run may not differ to that without learning, but new experiences will be stored in the agent's memory. Fig. 5 shows the learning run where the agent has its first interaction with the carrot. As the agent has no experience about the ability to satisfy its drives both, apple and carrot, are related to a cake that is stored in the agent's memory including a valuated experience to satisfy the hunger. So the agent thinks that both objects may satisfy the hunger in the same way as the cake would do. After having evaluated that the carrot doesn't meet its needs as expected, the agent stores the low satisfaction of the hunger. In the second simulation run the agent decides to take the apple which is more far away but evaluated as the better food source as the memories of the carrot from the 
learning simulation run. The agents then eat the apple instead of the carrot before (Fig. 6) showing a different behavior as in the first simulation run. In Table 1 an abstract view of the memories of the agents is shown. The memories changed as new experiences are stored after the agent perceived them.

\section{Conclusion and Outlook}

In reflection to its bodily needs, the agent learns in that way that experiences in fulfilling actions to satisfy its needs are stored to improve the decision making in a repeated similar situation. As we start in the primary process, the inspectors showed that the agent has no conscious knowledge in the second simulation run why he chooses the apple instead of the carrot. The higher valuation of the apple results in the experiences in the learning simulation run. This corresponds to the behavior of human beings who also can't give a reason why they choose sometimes this meal and sometimes a different food.

But they have also feelings related to the food which will be the next step in the integration of learning in the SiMA project. And not only feelings to objects, but also to situations, actions and perceived feelings from other agents can be useful to improve the own behavior of the agent.

\section{References}

[1] Dietrich, D. (2000). Evolution potentials for feldbus systems. Proceedings of IEEE International Workshop on $B d$ (pp. 145-146).

[2] Deutsch, T. (2011). Human Bionically Inspired Autonomous Agents -The Framework Implementation ARSi11 of the Psychoanalytical Entity Id Applied to Embodied Agents. Unpublished undergraduate dissertation, Vienna University of Technology.

[3] Luke, S., Cio-Revilla, C., Panait, L., \& Sullivan, K. (2004). Mason: A new multi-agent simulation toolkit. Proceedings of the Swarmfest Workshop. Retrieved from http://cs.gmu.edu/ eclab/projects/mason/publications/SwarmFest04.pdf

[4] Kandel, E. R. (1999). Biology and the future of psychoanalysis: A new intellectual framework for psychiatry revisited. American Journal of Psychiatry, 505-524.

[5] Freud, S. (1915). The unconscious, volume XIV on the history of the Psycho-Analytic movement. Papers on Metapsychology and Other Works. Vintag.

[6] Dietmar, D., Fodor, G., Zucker, G., \& Bruckner, D. (2009). Simulating the Mind -A Technical Neuropsychoanalytical Approach (1st ed.). Vienna: Springer.

[7] Jakubec, M., Doblhammer, K., Fittner, M., \& Wendt, A. (2015). Logical thought based on word presentations. Proceedings of EAPCogSci, EuroAsianPacific Joint Conference on Cognitive Science: Vol. 25-27. ( pp. 95-100).

[8] Wendt, A., Gelbard, F., Fittner, M., Schaat, S., \& Jakubec, M. (2015) Decision-making in the cognitive architecture siMA. Proceedings of Conference on Technologies and Applications of Artificial Intelligence.

[9] Franklin, S., Madl, T., D'Mello, S. \& Snaider, J. (2014). LIDA: A systems-level architecture for cognition, emotion, and learning. Proceedings of IEEE Transactions on Autonomous Mental Development: Vol. 6(1) (pp. $19-41)$.

[10] Velik, R. (2008). A Bionic Model for Human-like Machine Perception. Unpublished undergraduate dissertation, Vienna University of Technology, Institute of Computer Technology.

[11] Dietrich, D., Jakubec, M., Schaat, S., Doblhammer, K., Fodor, G., \& Brandstaetter, C. (2016). The fourth outrage of man (is the turing-test still up to date?). Journal of Computers, 12(2), 116-126.

[12] Atkinson, R. C., \& Shiffrin, R. M. (1968). Human memory: A proposed system and its control processes. In Spence, K. W., \& Spence, J. T. The psychology of learning and motivation, 2, 89-195. New York: Academic Press.

[13] Dietrich, D., Brandstätter, C., Doblhammer, K., Fittner, M., Fodor, G., Gelbard, F., Huber, M., Jakubec, M., Kollmann, S., Kowarik, D., Schaat, S., Wendt, A., \& Widholm, R. (2015). Natural scientific, psychoanalytical model of the psyche for simulation and emulation. Vienna University of Technology, Institute of Computer 
Technology. Retrieved from http://publik.tuwien.ac.at/files/PubDat_240983.pdf

[14] Schaat, S., Kollmann, S., Zhukova, O., Dietrich, D., \& Doblhammer, K. (2015) Examination of foundational AGI-agents in artificial-life simulations. Proceedings of Conference on Technologies and Applications of Artificial Intelligence (pp. 344-351).

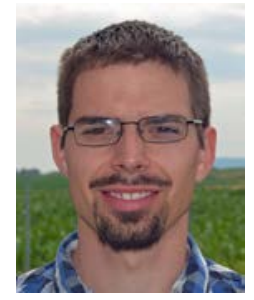

Martin Fittner was in born 1984 in Mistelbach, Austria, studied embedded systems at the University of Applied Science Technikum Wien in Vienna and finished with the degree master of science.

He works as a senior developer for software and system design for automotive electronic control units at the Robert Bosch AG. His main topics are testing software for factory testing, customer bootloader programming and security protection of the system. He is a member of the SiMA (Simulation of the Mental Apparatus and Applications) team. Currently, he works on his dissertation at the Vienna University of Technology in the field of the SiMA project.

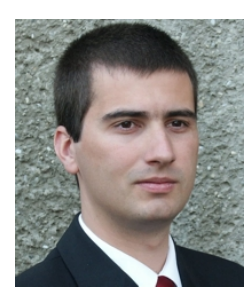

Christian Brandstätter was born in 1979 in Tulln, Austria, studied informatics and informatics-management at the Vienna University of Technology and finished both studies with the degree master of science.

He works as system administrator for general IT in a pharmaceutical research institute. His responsibility there is the IT infrastructure (network, storage and virtualization). Currently, he works on his dissertation and is a member of the Institute of Computer Technology at the Vienna University of Technology. Brandstätter is a member of IEEE. 